\title{
ARTÍCULOS
}

\section{Habilitación y obsolescencia de marcadores discursivos}

\author{
Developement and obsolescence of discursive markers
}

\author{
Emilio Ridruejo \\ Universidad de Valladolid \\ ridruejo@fyl.uva.es
}

\begin{abstract}
The starting hypothesis of the article is that the discursive operators of Spanish have not been enabled by grammaticalization processes identical to those of other grammatical components (such as verbal or nominal morphemes or prepositions and conjunctions), since there has been no reduction in the functional scope nor a total bleaching, so that its link with the original lexical items has not disappeared. The consequence is that markers are not integrated into paradigms as closed as grammatical paradigms and both their obsolescence and their renewal, therefore, follow similar patterns to what happens in the evolution of the lexicon. This hypothesis is tried to verify in the article by means of the examination of some discursive markers that, having been used diachronically in Spanish, have been abandoned in a relatively short time.
\end{abstract}

Keywords: discourse markers, grammaticalization, functional change, pragmatic change

1. En este artículo deseamos examinar si la habilitación de marcadores discursivos se ajusta a los mismos parámetros de los procesos de gramaticalización de elementos funcionales y, por tanto, también lo hace su evolución y su desaparición. Para ello, se han contrastado algunas de las características que se dan en la habilitación de marcadores con las de los procesos de gramaticalización de los elementos de relación. Tras formular una hipótesis que justifique las diferencias existentes en el blanqueo de los componentes semánticos y en la inclusión en paradigmas cerrados, se ha buscado contrastarla mediante el examen de la evolución de algunos marcadores caídos en desuso. 
En lo que atañe a los procesos de creación o habilitación de marcadores a partir de elementos de otras categorías se ha tratado ampliamente si se trata o no de gramaticalización (Traugott 1995; Estelles Arguedas 2009a: 28-32; Onodera 2011) o de pragmatización (Diewald 2011: 455-461). De hecho, las posiciones teóricas sobre cómo clasificar la creación de operadores discursivos dependen, como han mostrado Degand y Evers-Vermeul (2015), de lo que los investigadores entiendan como componentes de la gramática, y de la amplitud que estén dispuestos a conceder a los procesos de gramaticalización.

Ciertamente hay propiedades de los procesos de gramaticalización que se dan igualmente en los marcadores discursivos ${ }^{1}$. Estos quedan inmovilizados morfológicamente. No sería posible la moción de género o de número en marcadores como bueno o claro y la moción de tiempo y persona está muy fuertemente restringida en mira, oye (Pons Bordería 1998: 218). También la inmovilidad sintagmática, que supone una posición fija en la cadena hablada, se produce en gran parte de los marcadores discursivos del español. No obstante, aquellos marcadores que ejercen funciones en la interlocución suelen encabezar un enunciado y los que desempeñan funciones relacionales aparecen necesariamente en el margen inicial de una oración. En cambio, los marcadores discursivos son ajenos a lo que se considera el comportamiento más específico de los elementos gramaticales, esto es su ligazón (bondedness) con otro elemento léxico (Lehmann 1995: 148). En los marcadores discursivos solo se presenta (si es que lo hace) en forma de una mayor integración interna con fusiones de sintagmas complejos. Es lo que tiene lugar en ejemplos como otrossi, aosadas, etc., en los que se funde una conjunción y un verbo o una preposición y un adjetivo.

La reducción fónica, otro fenómeno que se produce en los procesos de gramaticalización (Lehman 1995: 126-127), falta habituaalmente en los marcadores discursivos. Si, excepcionalmente se produce univerbación, como en otrossi, aosadas, quia (Herrero Ruiz de Loizaga 2014), esta ha tenido lugar en etapas temporalmente muy próximas, de suerte que su evolución fónica no ha sido muy avanzada. En los procesos de gramaticalización cambia también el ámbito de inserción estructural de

\footnotetext{
${ }^{1}$ De las diferentes clasificaciones que se han establecido para los marcadores discursivos (Portoles 1998; Duque 2016: 50-52), a efectos prácticos de este trabajo, seguiremos solo la que establece tres grandes grupos: en primer lugar, los marcadores de estructuración y los de foco; junto a ellos los de modalidad y, en tercer lugar, los marcadores de interacción entre los hablantes (Loureda Lamas y Acín Villa 2010: 24). Puesto que las explicaciones diacrónicas han de proceder fundamentalmente de textos escritos, en ellos los marcadores reformuladores, los estructuradores del discurso y los de foco se documentan sin gran dificultad y se puede seguir su historia. Otros marcadores, en cambio, los que modulan la interacción entre hablantes, por su naturaleza se excluyen de la escritura, aunque puedan aparecer en algunos textos que, en ocasiones, intentan reproducir la expresión conversacional. Los marcadores de modalidad, finalmente, ocupan una posición intermedia en cuanto a su aparición en la documentación escrita.
} 
manera que unidades que funcionan autónomamente quedan integradas en otras y pasan a perder su función en el ámbito del que proceden (Lehmann 1995: 143-147; Tabor \& Traugott 1998). Sin embargo, como señalan Loureda Lamas y Acín Villa (2010: 43-44), en la habilitación de marcadores sucede lo contrario: un constituyente que se convierte en marcador discursivo, no solo no reduce su alcance estructural, sino que asume funciones que atañen a unidades más extensas de las originarias. De desempeñar funciones en el ámbito de una oración, pasan a ejercer funciones discursivas, englobando varias oraciones.

2. Uno de los mecanismos mejor descritos con respecto a la gramaticalización es que este proceso conlleva lo que se denomina blanqueo (bleaching), esto es, la eliminación del significado conceptual dirigido a establecer una referencia (Lehmann 1995: 127-131) y a adquirir una mayor subjetividad, frecuentemente formulada como una mayor implicación del emisor. Frente al blanqueo de los procesos de gramaticalización, se ha apuntado que los marcadores "en contadas ocasiones se desvinculan del contenido conceptual" (Garcés Gómez 2008: 207; Villar Díaz 2013: 167). En su habilitación se produce un cambio semántico en lo que se ha denominado mayor subjetivización, lo que implica un significado más abstracto (Company 2004a, 2004b) que afecta a los participantes del intercambio comunicativo o a su presentación del mensaje. De esta manera, mira no implica un acto directivo de percepción sensible o intelectual, o claro no alude a una determinada gama de intensidad lumínica. Se produce una especialización semántica: se aplican algunos de los rasgos semánticos a la producción discursiva o al discurso en sí para, a partir de tal especialización, dar lugar a determinadas inferencias que guíen la interpretación. Los marcadores que incluyen una base léxica fin, por fin, en fin, conservan rasgos semánticos de la base: en fin remite a la conclusión de una argumentación; por fin al término de un proceso que consuma espectativas previamente creadas (Garcés Gómez 2014: 93). Algo similar acaece con los marcadores de modalidad construidos a partir de predicados que califican un componente textual: claro, bueno (Martín Zorraquino 1994a, 1994b). La calificación el discurso mediante la cualidad formulada ha de restringirse, también metonímicamente, a la validez de la aserción. Igualmente es posible mostrar la vinculación que existe en los marcadores de contacto entre interlocutores con sus bases conceptuales. Algunos se fundan en predicados de percepción sensible o intelectual, formulados en imperativo, que dan lugar a actos directivos que recaen precisamente sobre el contacto: mira, oye, ¿sabes? (Pons Bordería 1998: 215; Iliescu 2014); otros, como el italiano prego, son verbos realizativos con una función parecida (Ghezzi, Molinelli 2014). Todos estos marcadores se utilizan configurando actos de habla que implican al interlocutor lo que conlleva, como indican Traugott y Dasher (2002: 19-23), un proceso de intersubjetivización, pero en todos ellos su significado léxico sigue parcialmente vigente, aunque no suponga ya estrictamente un sentido conceptual. En suma, en los marcadores discursivos no hay blanqueo, al menos no en el mismo 
grado que en los procesos de gramaticalización, sino una especialización y ello supone que, en la mayoría, las vinculaciones con el paradigma léxico no desaparecen por completo.

Según Traugott y Dasher (2002: 34-39), no hay un cambio pleno de significado en tanto en cuanto el significado codificado original sigue siendo accesible a los hablantes mediante el recurso a algún tipo de inferencia. Y en la habilitación de marcadores tales inferencias parecen resultar imprescindibles, pues es la acomodación al contexto lo que da lugar a su nuevo funcionamiento.

3. El paso último y más importante de un cambio de significado consiste en el proceso de integración del elemento gramatical en un paradigma (Lehmann 1995: 132-134). En virtud de la paradigmatización unos miembros del paradigma se oponen funcionalmente a los otros, con los cuales, en consecuencia, no pueden coincidir sintagmáticamente.

Con respecto a los marcadores discursivos se ha aceptado que, como los componentes gramaticales, se integran en paradigmas (Estellés Arguedas 2009a: 175 y ss.; Pons Bordería 2014; Garcés Gómez 2014: 165). Desde luego, no todos los marcadores se comportan de la misma manera. Para evaluar el grado de clausura de una estructura gramatical existe el recurso de examinar si existe la posibilidad de conmutación entre sus elementos. En los elementos de relación, preposiciones o conjunciones: hay conmutación, por ejemplo, aunque vino / puesto que vino implica una interpretación concesiva frente a la causal. En consecuencia, no pueden aparecer simultáneamente en el mismo nivel dos unidades que se oponen en un paradigma: *aunque, puesto que vino. Los marcadores discursivos no son exactamente comparables con esos elementos de relación. Es cierto que en algunas clases de marcadores discursivos, entre los estructuradores del discurso, por ejemplo, sí cabe defender la existencia de oposiciones: en primer lugar / además (Garcés Gómez 2014: 35). Pero es más dificil aceptar tales oposiciones para los marcadores de modalidad y para los de contacto. Por eso, varios marcadores de estos grupos pueden emplearse simultáneamente, sin que cambie la función de ninguno de ellos: oye, mira, claro, bueno, etc.

Si no hay paradigmas cerrados, la habilitación de un nuevo marcador no implica la reestructuración del paradigma, ni tampoco el cambio funcional de otros de sus componentes. La habilitación de o sea no va acompañada del desuso de es decir o de esto es y tampoco parece haber afectado, ni a las funciones de los dos últimos marcadores, ni a las variedades textuales en que se utilizan. Todavía más claro es el caso de la desaparición del evidencial dizque en el español peninsular (Garcés Gómez 2013: 293-297). No se puede suponer que haya sido resultado de un desplazamiento por la introducción en el paradigma de otro marcador de la misma función y, por ende, tampoco es posible explicar la razón de su permanencia en el español de América (Barraza Carbajal 2014: 3077-3083). La conclusión a la que hay que llegar es que numerosos marcadores se comportan como los componentes del vocabulario, cuyo inventario puede ampliarse o reducirse. 
4. En resumen, los marcadores discursivos ocuparían una posición intermedia entre los elementos de relación y los componentes del vocabulario y no se incorporan todos a paradigmas cerrados. Si no es preciso modificar las relaciones de todo un conjunto de unidades equifuncionales, será más fácil su evolución: mientras que es difícil que los paradigmas gramaticales se amplíen con nuevos componentes y, si esto sucede, su proceso de gramaticalización es muy lento, en cambio, una parte importante de los marcadores discursivos, incluso de los pertenecientes a los grupos más estructurados, se han habilitado, según la documentación, muy recientemente, algunos en épocas tan tardías como el siglo XVIII o el XIX. El inventario de Gregorio Garcés de 1791 (Martín Zorraquino 1998) nos proporciona datos sobre la modernidad de algunos marcadores discursivos. En ese inventario faltan marcadores como a lo mejor, desde luego, efectivamente, en resumidas cuentas, vale, allá tú, claro, oye, o sea, por cierto, a propósito, entre otros. Marcadores, como por cierto o ciertamente, aunque parecen tener función argumentativa ya en el siglo XIV (Villar Díaz 2013), solo son claramente discursivos en el siglo XVI. Otros, como por lo visto, que remite al origen de la información, solo se consolidan en el siglo XIX (Garcés Gómez 2013: 300-302).

La mayor parte de los estudios diacrónicos sobre los marcadores, por no decir todos, han recaído sobre los procesos de creación (Pons Rodríguez 2010), pero no se ha estudiado su decadencia, que se presume muy rápida. Algunos de los marcadores documentados en textos medievales o clásicos son hoy desconocidos o inusitados. Podemos citar otrossi o ahé. El primero es utilizado extensamente en los siglos XIII y XIV, pero posteriormente queda fosilizado en la lengua jurídica (Espinosa Elorza 1995); el empleo de ahé queda reducido a las traducciones bíblicas, aunque quizá fundido con el derivado de AD FIDEM, se mantiene en español clásico.

5. Sucede, no obstante, que frecuentemente los cambios que les afectan se han querido explicar como consecuencia de las relaciones que se dan en el seno de un mismo paradigma (Pons Bordería 2014: 124-135). Sin embargo, la hipótesis de la presión paradigmática ha sido utilizada de manera contradictoria en los estudios sobre marcadores, unas veces para dar cuenta la atracción semántica y funcional que ejerce un paradigma sobre un marcador, o sea que, por cierto, para que llegue a integrarse en él, otras, al contrario, suponiendo que un marcador es desplazado por la existencia en el paradigma de otros marcadores equivalentes.

En realidad, de acuerdo con lo indicado con respecto a la ausencia de paradigmas cerrados, la decadencia o la desaparición comprobadas de ciertos marcadores no implica necesariamente su reemplazo por otros funcionalmente equivalentes y tampoco la incorporación de nuevos marcadores conlleva necesariamente la desaparición de otros preexistentes. Otrossi como un marcador de adición empleado en textos administrativos y judiciales no ha sido sustituido por otro de la misma función: o bien se emplean otros marcadores como además (que no es exactamente equi- 
valente funcional), o bien la mera estructura textual basta para señalar la adición de un nuevo elemento. $\mathrm{Y}$ es que, al igual que sucede con el léxico, dado que este se configura en series ilimitadas, una nueva incorporación no borra otros elementos y, en consecuencia, no es imprescindible la adaptación de todo un conjunto de relaciones funcionales en el interior de un paradigma. Y si la presión paradigmática no es decisiva en la evolución de los marcadores discursivos, entonces los cambios de cada elemento han de ser considerados como autónomos.

6. Se pueden presentar algunos casos concretos de mecanismos de habilitación y de desuso de marcadores discursivos. Aosadas es un marcador cuya evolución ha sido trazada con detalle por Ricós Vidal (2008). Inicialmente se trata de una locución adverbial, compuesta con la preposición $a$ más un adjetivo en -as o un participio con función adjetival. Su sentido, como en muchas locuciones similares, es el de una locución adverbial de modo (idéntico a otras locuciones como a oscuras, a derechas, etc. $)^{2}$. El giro modifica a todo un predicado y significa originalmente 'de manera arriesgada, osada': «Aosadas corred, que por miedo non dexedes nada, / Fita ayuso e por Guadalfajara» (Cantar de Mio Cid, ed. de Ramón Menéndez Pidal, Madrid, Espasa Calpe, 1964: 443). Posteriormente, ya en su función discursiva, aosadas se emplea para calificar como arriesgada, esto es incierta o insegura, una determinada proposición. Funciona, por tanto, como un marcador de modalidad y este es probablemente el uso que le da López de Ayala: «O en Abenverga podedes, si quisierdes, ser librado, / o en Aben- caçi, aosadas, arrendador del bispado, / o en diezmos de la mar, que es dinero bien contado: / escojed dó vos quisierdes, luego seredes pagado» (Canciller Lopez de Ayala, Libro rimado de Palaçio, ed. de Jacques Joset, Madrid, Alhambra, 1978: 468b).

Resulta, así, que la proposición modificada por aosadas se formula como arriesgada para el emisor. Sin embargo, a partir de tal suposición, el emisor, solo por hacerla, suscribe un compromiso y con ello enfatiza la aseveración. El mismo hecho de que se produzca la calificación de arriesgada contradice o atenúa la percepción de incertidumbre, pues, en realidad, implica que el emisor tiene razones suficientes para enunciar la proposición. Es decir, antitéticamente, aosadas pasa a significar 'con certeza, con seguridad' y es, por tanto, un marcador de refuerzo de la aserción. Este es exactamente el empleo que se documenta a fines del siglo XV en La Celestina y que también explica Correas: «CELESTINA.-¡Ay como huele toda la ropa en bulléndote! ¡A osadas, que está todo a punto!» (Fernando de Rojas, La Celestina, ed. de Humberto López Morales, Madrid, CUPSA, 1976: 122). «Aosadas, ke kien lo dixo no mintió. Dízese enkareciendo algo ke kunplidamente se dixo o hizo; kasi lo

\footnotetext{
${ }^{2}$ Nebrija en su Gramática castellana recoge esta locución junto a otras semejantes: «Otros dezimos por rodeo desta preposicion. a. i de algun nombre como apenas aosadas asabiendas adrede» (Nebrija 1946 [1492]: 85).
} 
mesmo ke: Ahotas $^{3}$ : konfiadamente. Dicho komo bordonzillo. Algunos: Asuadas» ${ }^{4}$ (Correas 1967: 32 y 603-604).

7. Si aosadas se habilita como marcador en virtud de un cambio semántico y funcional concreto, en otras ocasiones, el empleo de un marcador discursivo puede ser resultado de un préstamo, asociado o no a un determinada tradición discursiva. Según Morby (1968: 191, nota 150) tal mecanismo se da en cosa que construido con subjuntivo. En este giro que no es relativo, sino la conjunción de subordinación (de otra manera no se explicaría el subjuntivo) y, en tal caso, resulta de una elipsis de "No sea cosa que...", en la que el verbo ser en subjuntivo funciona como un predicado creador de mundos, con polaridad negativa. Como marcador discursivo se usa para indicar que la proposición afectada ha de ser tomada como una conjetura. Puede interpretarse, por tanto, también como un operador de modalidad, tal como aparece en los textos siguientes: «GER. Y a fe que quien pasó a tales horas, que no venía a burlar. Toribio dijo: "Montañés será tu marido". CEL.¿Cosa que sea destos que venden agua?» (Lope de Vega, La Dorotea, ed. de Edwin S. Morby, Madrid, Castalia, 1968: 203).

«Buena es la conversación; / que es éste don Luis ignora. / ¡Cosa que le diese agora» (Rojas Zorrilla, Entre bobos anda el juego CORDE).

La mayor parte de los ejemplos de cosa que aparecen documentados en los textos de Lope de Vega o de autores de su escuela. Morby (1968: 191, nota 150) cita otros ejemplos de Lope recogidos en obras como La pobreza estimada, III, 161; El acero de Madrid, III, 385b; El galán de la membrilla, II, 105b; Sin secreto no hay amor, III, 163b, etc.

8. Las dos formas, aosadas y cosa que utilizados como marcadores desaparecieron como tales con rapidez. Aosadas tiene empleos escasos ya en el siglo XVI y a pesar de la descripción de Correas, los últimos ejemplos que aporta el CORDE son de 1605, de López de Úbeda. Por tanto, no tenemos documentación segura como marcador más que durante poco más de un siglo ${ }^{5}$. Con respecto a cosa que como marcador de modalidad no se encuentra en CORDE con posterioridad a 1640.

\footnotetext{
${ }^{3}$ Ahotas, tal como indica Correas, tiene una suerte y una función semejante a aosadas. Se trata de una formación adverbial, quizá derivada de hoto < FATUM, que se emplea igualmente como marcador de modalidad. Según el estudio de Ricós Vidal (2012: 69) su documentación es casi exclusiva de la lengua de la comedia pastoril del siglo XVI (de Gil Vicente, Juan de la Encina, Lucas Fernández) y de autores que la imitan.

${ }^{4}$ La variante Asuadas, que recoge Correas, no solo presenta la fusión de la preposición, sino que hay metátesis que hace más opaca la relación con el étimo.

${ }^{5}$ Ricós Vidal (2008) recoge un ejemplo del erudito Bartolomé José Gallardo de 1836: «Medio Bruja asmo que es: y aun, a osadas, que si buscarla querrés, cadalnoche la topé» (Bartolomé José Gallardo, El Criticón. Número Cuarto, página II, 25, CORDE). Se trata, sin duda, de un uso mediante el cual el autor procura imitar el habla rústica, dado que en el mismo texto aparecen otras formas igualmente desusadas y marginales (cadalnoche, diabro, afrita, etc.). Puede suceder con los marcadores discursivos, al igual que con items léxicos, que caigan en desuso en la lengua general, pero que persistan
} 
En ambos casos (como en otros) se hace preciso formular una hipótesis acerca de una utilización tan efímera. En la evolución de los marcadores discursivos, tal como se señala arriba, se mantiene una vinculación semántica con los componentes léxicos de los que proceden. Pues bien, puede suceder que, por la evolución del marcador o por algún cambio en la base orginaria, se vuelva opaca la relación. En tal caso, también queda dificultada la función específica del marcador como guía de inferencias para el destinatario, puesto que la función discursiva deriva y es una especialización de ese inicial significado conceptual.

Estos procesos parecen haber tenido lugar con aosadas y con cosa que. Aosadas como marcador discursivo tiene un sentido que ha quedado enteramente desligado del de la base sobre la que se formó: osar. El cambio de tipo antitético sobre el que se funda su función como marcador lo aleja precisamente del significado de sus componentes iniciales haciéndolo opaco. Y el mismo proceso de opacificación se repite en el otro ejemplo que hemos mostrado. Es posible que la rapidez de la habilitación de cosa que haya sido resultado de un préstamo, pero su desuso puede deberse también a la opacidad del marcador: se ha constituido a partir de la formulación de una hipótesis que se funda en el verbo ser, pero precisamente la elipsis de este verbo es lo que vuelve opaco el marcador.

¿Es imprescindible que la opacidad de un marcador, entendiendo por tal la desvinculación con los items léxicos de los que procede, produzca necesariamente su obsolescencia? Debe quedar claro que no. Ningún cambio lingüístico está determinado obligatoria y mecánicamente. Hay marcadores que están vivos, aunque no exista ningún significado descriptivo. Es el caso, entre otros, de los marcadores de recepción, ah (Vázquez Vega 2003: 172 y ss.), de contacto, che en español de Valencia y de los países del Plata, y también cáchai en español de Chile. Los dos primeros son, seguramente, lo que se denominan voces naturales (García de Diego 1968: 111 y 206) y el último, quizá, un indoamericanismo. Su funcionamiento como marcadores queda, por tanto, al margen de cualquier vinculación inicial con otros elementos léxicos, pues nunca hubo para ellos significado referencial transparente.

\section{CONCLUSIONES}

Al contrastar la habilitación de marcadores discursivos con el desarrollo de los procesos de gramaticalización, se comprueba que en aquellos no se produce un blanqueo semántico pleno, de manera que los marcadores discursivos no se desvinculan totalmente de los items léxicos originarios. Además, la mayor parte de los marcadores discursivos tampoco quedan integrados en paradigmas cerrados $\mathrm{y}$, en consecuencia, no desplazan a otras unidades funcionalmente equivalentes.

en variadades dialectales arcaizantes. Ello explica que a osadas hubiera sido seleccionada por Gallardo como un rasgo arcaizante y también que a osadas siga vivo en el catalán de Valencia. 
A estas dos características de los marcadores discursivos responde al hecho de que su habilitación tiene lugar en un intervalo de tiempo mucho más reducido que el que se da en los procesos de gramaticalización y se explica porque los cambios funcionales que suceden no afectan a las relaciones de todo un conjunto de elementos, tal como acaece en los cambios gramaticales.

Estas propiedades también permiten explicar la razón por la que algunos marcadores discursivos caen en el olvido con mayor rapidez que los componentes de la gramática. Se ha examinado la evolución de algunos marcadores discursivos, aosadas y cosa que, y se ha dado cuenta de su rápida desaparición, que podría ser atribuida al oscurecimiento del vínculo semántico que los relaciona con los items de origen, de suerte que su opacidad dificulta las inferencias que pueden generar o, lo que es lo mismo, su funcionamiento como marcadores. 


\section{BIBLIOGRAFÍA}

Barraza Carbajal, G. (2014). Oraciones subordinadas sustantivas de objeto directo. In C. Company Company (ed.), Sintaxis histórica de la lengua española. Tercera parte: Adverbios, preposiciones y conjunciones. Relaciones interoracionales (capítulo 25, vol. 3). México: Universidad Nacional Autónoma de México. Fondo de Cultura Económina.

Company Company, C. (2004a). ¿Gramaticalización o desgramticalización? Reanálisis y subjetivización de verbos como marcadores discursivos in la historia del español. RFE, 84, 29-66.

Company Company, C. (2004b). Gramaticalización por subjetivización como prescindibilidad de la sintaxis. NRFH, 52, 1-27.

Correas, G. (1967). Vocabulario de refranes y frases proverbiales. Texte établi par L. Combet. Bordeaux: Féret et fils.

Degand, L. \& Evers-Vermeul, J. (2015). Grammaticalization or pragmaticalization of discourse markers? Journal of Historical Pragmatics, 16, 1, 59-85.

Diewald, G. (2002). A model for relevant types of contexts in grammaticalization. In I. Wischer \& G. Diewald (eds.), New Reflections on Grammaticalization (pp. 103-117). Amsterdam/Philadelphia: John Benjamins.

Diewald, G. (2011). Grammaticalization and pragmaticalization. In H. Narrog \& B. Heine (eds.), The Oxford Handbook of Grammaticalization (pp. 450-461). Oxford: Oxford University Press.

Duque, E. (2016). Las relaciones de discurso. Madrid: Arco/Libros.

Espinosa Elorza, R.M. (1995). Adverbios aditivos en la lengua medieval y clásica. Verba, 22, 585-594.

Estellés Arguedas, M. (2009a). Gramaticalización y gramaticalizaciones. El caso de los marcadores del discurso de digresión en español. Tesis doctoral. Valencia: Universitat de València. Obtenida en <http://roderic.uv.es/handle/10550/15767>.

Estellés Arguedas, M. (2009b). Un caso atípico de gramaticalización: el valor epistémico del marcador por cierto. RILCE, 25, 2, 319-339.

Garcés, G. (1852, 1791). Fundamento del valor y elegancia de la lengua castellana. Madrid: Rivadeneira.

Garcés Gómez, P. (2008). La organización del discurso: marcadores de ordenación y de reformulación. Madrid: Iberoamericana/Vervuert.

Garcés Gómez, P. (2013). La formación y evolución del paradigma de los operadores discursivos matizadores de la verdad del enunciado. In P. Garcés Gómez (ed.), Los adverbios con función discursiva: procesos de formación y evolución (pp. 275-316). Madrid: Iberoamericana/Vervuert.

Garcés Gómez, P. (2014). Diacronía de los marcadores discursivos y representación in un diccionario histórico. La Coruña: Universidade da Coruña.

García de Diego, V. (1968). Diccionario de voces naturales. Madrid: Aguilar.

Ghezzi, Ch. \& Molinelli, P. (2014). Deverbal pragmatic markers from Latin to Italian (Lat. QUAESO and It. prego). The cyclic nature of functional developments. In Ch. Ghezzi \& P. Molinelli (eds.), Discourse and Pragmatic Markers from Latin to the Romance Languages (pp. 61-85). Oxford: Oxford University Press.

Herrero Ingelmo, J.L. (2006-2007). ¿Cómo surgen los conectores: los reformuladores del discurso id est, esto es, es decir? Revista de Lexicografia, 13, 45-54.

Herrero Ruiz de Loizaga, F.J. (2014). Quia, ca, qué va. Elipsis y gramaticalización de elementos interjectivos de negación. In J.L. Girón Alconchel \& D.M. Sáez Rivera (eds.), Procesos de gramaticalización in la historia del español (pp. 233-262). Madrid: Iberoamericana/Vervuert. 
Hopper, P.J. (1991). On some principles of grammaticization. In E.C. Traugott \& H. Bernd (eds.), Approaches to Grammaticalization (vol. I, pp. 17-36). Amsterdam/Philadelphia: John Benjamins.

Iliescu, M. (2014). Call markers in French, Italian, and Romanian. In Ch. Ghezzi \& P. Molinelli (eds.), Discourse and Pragmatic Markers from Latin to the Romance Languages (pp. 29-40). Oxford: Oxford University Press.

Lehmann, Ch. (1995). Thoughts on Grammaticalization. München: LINCOM Europa.

Loureda Lamas, Ó. \& Acín Viña, E. (eds.) (2010). Los estudios sobre marcadores del discurso en español, hoy. Madrid: Arco/Libros.

Martin Zorraquino, M.A. (1994a). Algunas observaciones sobre claro como operador pragmatico en español actual. In G. Hilty (ed.), Actes $d u X X^{e}$. Congres International de Linguistique et Philologie Romanes, vol. 1 (pp. 467-78). Tübingen: Francke.

Martin Zorraquino, M.A. (1994b). Bueno como operador pragmatico in español actual. In A. Alonso et al. (eds.), II Encuentro de linguistas y filologos de Espana y Mexico (pp. 402-412). Salamanca: Universidad de Salamanca.

Martin Zorraquino, M.A. (1998). Dos notas a proposito del Tratado de las particulas de G. Garces (1791). Un precedente in el estudio de los marcadores del discurso. In E. Ramon Trives \& H. Provencio Garrigos (eds.), Estudios de lingüistica textual. Homenaje al Profesor Muñoz Cortes (pp. 307-315). Murcia: Universidad de Murcia.

Martín Zorraquino, M.A. \& Portolés, J. (1999). Los marcadores del discurso. In I. Bosque \& V. Demonte (eds.), Gramática descriptiva de la lengua española, vol. III (pp. 4051-4213). Madrid: Espasa Calpe.

Morby, E.S. (1968). Lope de Vega. «La Dorotea». Edición de Edwin S. Morby. Madrid: Castalia.

Nebrija, A. (1946 [1492]). Gramática Castellana. Edición crítica de P. Galindo Romeo y L. Ortiz Muñoz. Madrid: Edición de la Junta del Centenario.

Onodera, N.O. (2011). The Grammaticalization of Discourse Markers. In H. Narrog \& B. Heine (eds.), The Oxford Handbook of Grammaticalization (pp. 614-624). Oxford: Oxford University Press.

Pons Bordería, S. (1998). Oye y mira o los límites de la conexión. In M.A. Martín Zorraquino \& E. Montolío Durán (eds.), Marcadores del discurso. Teoría y análisis (pp. 213-228). Madrid: Arco/Libros.

Pons Bordería, S. (2008). Gramaticalización por tradiciones discursivas: el caso de esto es. In J. Kabatek (ed.), Sintaxis histórica del español y cambio lingüistico. Nuevas perspectivas desde las Tradiciones Discursivas (pp. 249-274). Madrid/Frankfurt: Iberoamericana/Vervuert.

Pons Bordería, S. (2014). Paths of grammaticalization in Spanish o sea. In Ch. Ghezzi \& P. Molinelli (eds.), Discourse and Pragmatic Markers from Latin to the Romance Languages (pp. 109-136). Oxford: Oxford University Press.

Pons Rodríguez, L. (2010). Los marcadores del discurso in la historia del español. In O. Loureda Lamas \& E. Acín Villa (eds.), Los estudios sobre marcadores del discurso in español, hoy (pp. 523-616). Madrid: Arco/Libros.

Portolés, J. (1998). Marcadores discusivos. Barcelona: Ariel.

Real Academia Española, Corpus diacrónico del español (CORDE). Banco de datos CORDE [in línea], $<$ http.//www.rae.es $>$.

Ricós Vidal, A. (2008). Locuciones adverbiales con preposición a y adjetivo in el español y el portugués del siglo XVI. Estudio histórico. In Actas del VII Congreso Internacional de la Lengua Española. Mérida (Yucatán). 4-8 de septiembre de 2006 (pp.1035-1054). Madrid: Arco/Libros. 
Ricós Vidal, A. (2012). A reculas, a hotas, a sabiendas, a la deshilada, a la callada, a la sorda: más sobre locuciones adverbiales. In A. García Valle et al. (eds.), Fablar bien e tan mesurado: veinticinco años de investigación diacrónica in Valencia: estudios ofrecidos a M. Teresa Echenique Elizondo in conmemoración de su cátedra (pp. 68-86). Valencia: Tirant Humanidades.

Tabor, W. \& Traugott, E.C. (1998). Structural scope expansion and grammaticalization. In A. Giancalone Ramat \& P.J. Hopper (eds.), The Limits of Grammaticalization (pp. 229-272). Amsterdam/Philadelphia: John Benjamins.

Traugott, E.C. (1995). The role of grammaticalization in the development of discourse markers. Paper presented in ICHL XII Manchester. Disponible en: http://www.stanford.edu/ traugott/ papers/discourse.pdf.

Traugott, E.C. \& Dasher, R.B. (2001). Regularity in Semantic Change. Cambridge: Cambridge University Press.

Traugott, E.C. \& Heine, B. (eds.) (1991). Approaches to Grammaticalization. Focus on Theoretical and Methodological Issues. Amsterdam/Philadelphia: John Benjamins.

Vázquez Vega, N. (2003). Marcadores discusivos de recepción. Santiago de Compostela: Universidad de Santiago de Compostela.

Villar Díaz, M.B. (2013). La evolución de los adverbios y locuciones adverbiales de modalidad epistémica. In P. Garcés Gómez (ed.), Los adverbios con función discursiva: procesos de formación y evolución (pp. 157-199). Madrid: Iberoamericana/Vervuert. 